(2) Open Access Full Text Article

\title{
Superselective intra-arterial melphalan therapy for newly diagnosed and refractory retinoblastoma: results from a single institution
}

This article was published in the following Dove Press journal:

Clinical Ophthalmology

24 May 2013

Number of times this article has been viewed

\author{
Sheila Thampi' \\ Steven W Hetts ${ }^{3}$ \\ Daniel L Cooke ${ }^{3}$ \\ Paul J Stewart ${ }^{2}$ \\ Elizabeth Robbins' \\ Anuradha Banerjee' \\ Steven G DuBois' \\ Devron Char ${ }^{2}$ \\ Van Halbach ${ }^{3}$ \\ Katherine Matthay' \\ 'Department of Pediatrics, \\ ${ }^{2}$ Department of Ophthalmology, \\ ${ }^{3}$ Department of Radiology and \\ Biomedical Imaging, Division of \\ Neurointerventional Radiology, \\ University of California, San Francisco \\ School of Medicine, San Francisco, \\ CA, USA
}

Background: Intra-arterial administration of melphalan chemotherapy has shown promise in the treatment of retinoblastoma. This report describes our results using superselective intraarterial melphalan in patients with newly diagnosed retinoblastoma and those who were treated for progression after systemic chemotherapy.

Methods: This is a retrospective review of all retinoblastoma patients treated with intra-arterial melphalan at the University of California, San Francisco from March 2010 to August 2012. Twenty eyes (16 patients) underwent 40 intra-arterial melphalan infusions, and dose was determined by age. Patients were treated at monthly intervals and received a range of $1-5$ treatments. Response to therapy, toxicity, and procedural radiation exposure was assessed.

Results: All patients are alive without metastatic disease at a median follow-up of 14.5 (1-29) months. Treatment with enucleation or external beam radiation was avoided in 11/20 eyes $(55 \%)$ overall [6/12 (50\%) in newly diagnosed eyes and 5/8 (63\%) in refractory/relapsed eyes]. Response rates (per the International Classification of Retinoblastoma) were as follows: 6/7 (86\%) in groups A-C and 5/13 (38\%) in groups D and E. Nonhematologic and hematologic toxicities were minimal and comparable with those in previous reports. The mean procedural radiation dose was $20.2 \pm 11.9 \mathrm{mGy}$ per eye per procedure.

Conclusion: Superselective intra-arterial melphalan therapy is effective for less advanced eyes but further modifications to therapy are required to improve results in eyes with advanced retinoblastoma.

Keywords: retinoblastoma, intra-arterial, melphalan

\section{Introduction}

Retinoblastoma is the most common intraocular cancer of childhood, affecting approximately 300 individuals in the United States annually. ${ }^{1}$ It typically presents in children under the age of 5 years. ${ }^{1}$ In $25 \%$ of cases, the tumors involve both eyes. $^{2}$ Although the 5-year overall survival of retinoblastoma is $96.5 \%$ according to Surveillance, Epidemiology and End Results data for the years 1995-2004, there is a significant threat to useful vision, particularly in patients with bilateral disease. ${ }^{3}$ Furthermore, in patients with hereditary retinoblastoma, inactivation of the $R B 1$ gene in germline tissue predisposes patients to secondary malignancies. Patients with the hereditary form of retinoblastoma have a $36 \%$ cumulative incidence of secondary tumors at 50 years after diagnosis, whereas patients with sporadic retinoblastoma have only a $5.7 \%$ cumulative incidence. ${ }^{4}$ Radiation therapy further increases the risk of secondary cancer in patients who inherit the disease by as much as three-fold. ${ }^{4}$ Therefore, treatment goals, in addition to vision preservation and avoidance of
Correspondence: Katherine Matthay 505 Parnassus Avenue, M649, Box 0106, San Francisco, CA 94I43, USA

$\mathrm{Tel}+\mathrm{I} 4154760603$

Fax + I 4155024372

Email matthayk@peds.ucsf.edu 
enucleation, must include minimizing exposure to therapies that may further predispose to secondary malignancies.

Treatment options for retinoblastoma include local therapies, such as laser photocoagulation or cryotherapy, systemic intravenous chemotherapy, surgical enucleation, radiation therapy, and more recently, intra-arterial chemotherapy. Historically, patients with disease classified by the International Classification of Retinoblastoma as group A or B can be controlled with systemic chemotherapy and local therapies, but patients with very advanced or refractory/ relapsed disease may require enucleation or external beam radiation. ${ }^{5,6}$

Intra-arterial chemotherapy encompasses several techniques, which have evolved significantly since the first report of direct injection of triethylene melamine into the cervical internal carotid artery in $1958,{ }^{7}$ which also resulted in significant doses of intra-arterial chemotherapy affecting the ipsilateral brain. Direct puncture of the supraorbital artery or superficial temporal artery with retrograde perfusion of the eye was also described in the 1960s. ${ }^{8}$ By the 1990s, $\mathrm{x}$-ray fluoroscopic guidance of endovascular catheters had improved to the point that transfemoral arterial access (a safer approach than direct carotid or scalp artery puncture) allowed inflation of an endovascular balloon in the internal carotid artery above the origin of the ophthalmic artery and infusion of a chemotherapeutic drug (often melphalan) into the internal carotid artery such that most of the drug would selectively perfuse the ophthalmic artery. ${ }^{9,10}$ In the last decade, superselective techniques involving placement of very small microcatheters transfemorally into the ophthalmic artery itself have permitted chemotherapeutic infusions to the eye with minimal dose distribution elsewhere. ${ }^{11,12}$

The potential benefits of superselective intra-arterial chemotherapy are avoidance of enucleation, preservation of vision, prevention of secondary malignancies associated with high-dose radiation therapy, and limiting both acute and late toxicities of high-dose systemic chemotherapy. There is still only limited knowledge of the acute and late toxicities associated with intra-arterial therapy, including the long-term effect on vision. Furthermore, possible late effects of radiation exposure from x-ray fluoroscopy during intra-arterial infusion catheter placement have not been fully elucidated.

Several studies have been conducted, primarily in patients with refractory retinoblastoma. These studies have demonstrated that intra-arterial therapy can be used with success in combination with other therapies to prevent enucleation in patients with refractory disease. ${ }^{13}$ The use of intra-arterial chemotherapy in patients with newly diagnosed retinoblastoma has been less frequently reported, but results show a better response to intra-arterial chemotherapy in newly diagnosed eyes as compared with previously treated eyes. ${ }^{14}$ This report summarizes our results using superselective intra-arterial melphalan in selected patients with newly diagnosed ocular retinoblastoma as well as in those with refractory/relapsed retinoblastoma.

\section{Materials and methods}

All patients with ocular retinoblastoma treated with intraarterial melphalan therapy at the University of California, San Francisco (UCSF) from March 2010 to August 2012 were included in this report. The UCSF Committee on Human Research approved the review of patient medical records. Parental informed consent for intra-arterial melphalan was obtained prior to each procedure. Electronic and paper medical records as well as RetCam photographs were reviewed to collect information regarding demographics, diagnosis, initial tumor stage (based on International Classification of Retinoblastoma), initial treatment, response to intra-arterial therapy, use of concurrent local retinal therapy (laser or cryotherapy), additional therapy after intra-arterial chemotherapy, current disease/life status, and duration of follow-up. No patients had known metastatic disease at the start of intra-arterial treatment. Data and follow-up were updated through September 2012.

Ophthalmic artery melphalan infusions were performed by interventional neuroradiologists at UCSF. While under general anesthesia, femoral artery access was established and a baseline activated clotting time was obtained. In order to reduce potential thrombotic complications, intravenous heparin $(70 \mathrm{U} / \mathrm{kg}$ ) was used during the procedure, with systemic anticoagulation confirmed by repeat activated clotting time. Low-dose fluoroscopy settings were used during the procedure and diagnostic angiograms of the carotid artery were obtained using a 4 French diameter catheter. A 1.5-1.8 French microcatheter was then placed through the 4 French catheter, and the microcatheter was used to access the origin of the ophthalmic artery of the affected eye. Whereas digital subtraction angiograms were performed of the carotid artery and the ophthalmic artery in initial patients, in later patients, the diagnostic $\mathrm{x}$-ray dose was reduced by eliminating carotid angiograms (instead navigating catheters under fluoroscopic road mapping) and performing only ophthalmic artery angiograms to confirm perfusion of the entire retina via the microcatheter.

Melphalan was administered via the microcatheter into the ophthalmic artery with pulsatile hand injections over 
30 minutes, with one brief contrast injection under fluoroscopy 15 minutes into the infusion to confirm persistent perfusion of the retina. In patients undergoing bilateral intraarterial chemotherapy in a single session, the contralateral carotid and ophthalmic arteries were catheterized via the same femoral access and the intra-arterial chemotherapy infusion was repeated. When all intra-arterial chemotherapy infusions were completed, the catheter was removed, anticoagulation was reversed, and manual pressure was applied to the femoral access site for 15 minutes to obtain hemostasis. Patients recovered from anesthesia and were observed for 6 hours after the procedure before being discharged to home.

Doses of intra-arterial melphalan were based on patient age as per Gobin et al, ie, children aged 6-12 months were given $3 \mathrm{mg}$, those aged $1-3$ years were given $4 \mathrm{mg}$, and those older than 3 years were given $5 \mathrm{mg} .{ }^{14}$ When both eyes were treated at a single session, intra-arterial doses were reduced so as not to exceed $3 \mathrm{mg}$ in each eye. Criteria for additional intra-arterial therapy sessions were based on the response noted on ophthalmologic examination under anesthesia.

Patients had a complete blood count checked prior to treatment and then again approximately 10 days after each intra-arterial treatment to evaluate for myelosuppression. To determine disease response, an ocular oncologist performed an eye examination under anesthesia 3-4 weeks after intraarterial treatment using RetCam photographs. Cases were reviewed by ophthalmologists (DC, PS) to classify response to therapy. Best response to intra-arterial melphalan was categorized as decrease in size of tumor, no response, unable to evaluate response, and findings consistent with known regression patterns. Regression patterns include type 1 (cottage cheese/calcified regression), type 2 (fish flesh/noncalcified regression), type 3 (a combination of type 1 and 2), and type 4 (regression into a flat scar). Progression was defined as increasing size of tumor, appearance of new tumors, or new vitreous seeding.

\section{Results \\ Patient characteristics}

Table 1 shows the characteristics of the 16 patients who underwent intra-arterial melphalan therapy. The median age at the time of first intra-arterial treatment was 15 (7-63) months. There were 10 children who initially presented with bilateral disease, but only four of those patients had both eyes treated with intra-arterial melphalan. There were four patients with a known family history of retinoblastoma. Among the 16 patients, 20 eyes were treated with intra-arterial melphalan, with a range of 1-5 treatments per eye for a total of 40 sessions. Based on the International Classification for Retinoblastoma, there was one group A eye, four group B eyes, two group $\mathrm{C}$ eyes, 11 group $\mathrm{D}$ eyes, and two group $\mathrm{E}$ eyes at initial diagnosis. Patient 14 presented with bilateral retinal detachment and bilateral neovascularization of the iris, and was staged as bilateral group E retinoblastoma. Twelve eyes were newly diagnosed and intra-arterial therapy was the first form of treatment, while all refractory or relapsed eyes had a previous history of treatment with systemic chemotherapy and local measures $(n=8$ eyes). Previous local therapies included laser ablation ( $n=8$ eyes), with one eye receiving both laser ablation and cryotherapy. No patients received plaque therapy. Previous systemic chemotherapy included a range of 4-10 cycles of intravenous carboplatin, etoposide, and vincristine ( $\mathrm{n}=8$ eyes) with additional carboplatin and vincristine for three cycles, and cyclophosphamide and topotecan for three cycles in the same three eyes. No patients received external beam radiation therapy prior to intra-arterial melphalan.

\section{Treatment and outcomes}

Melphalan doses ranged from $3 \mathrm{mg}$ to $5 \mathrm{mg}$ per eye. All intra-arterial treatments were technically successful as defined by injection of melphalan in the ophthalmic artery. In patient 7 , the ophthalmic artery was accessed via the zygomatico-orbital branch of the left superficial temporal artery, which provided a collateral approach to the ophthalmic artery. Patient 12 also had successful access to the ophthalmic artery, but an anatomic variant was noted such that the meningolacrimal branch of the right middle meningeal artery was catheterized. Most patients received a melphalan dose based on age as described in previous reports, ${ }^{14}$ but doses for two patients were increased because of extensive retinal involvement and the dose in one patient was decreased because of heavy pretreatment with systemic chemotherapy.

Thirteen of the 20 eyes (65\%) received concurrent local eye therapy with laser ablation or cryotherapy. Six of 20 eyes $(30 \%)$ were enucleated due to disease progression after intra-arterial therapy and four of 20 eyes (20\%) received external beam radiation therapy after intra-arterial therapy. One eye received both radiation therapy and enucleation, such that a total of nine eyes required enucleation and/or radiotherapy despite intra-arterial treatment. Two eyes required systemic chemotherapy due to high-risk features: one patient was found to have high-risk features (choroidal and optic nerve invasion) in the non-intra-arterial treated eye and the other patient had imaging findings concerning 
Table I Patient and treatment characteristics

\begin{tabular}{|c|c|c|c|c|c|c|c|c|c|}
\hline Pt \# & $\begin{array}{l}\text { Initial eye } \\
\text { invol }\end{array}$ & $\begin{array}{l}\text { Age at } \\
\text { Dx (mo) }\end{array}$ & FH of RB & $\begin{array}{l}\text { ICRB at Dx } \\
\text { (OD/OS) }\end{array}$ & $\begin{array}{l}\text { Past local } \\
\text { Rx }\end{array}$ & $\begin{array}{l}\text { Past systemic } \\
\text { Rx (cycles) }\end{array}$ & $\begin{array}{l}\text { Eye Rx'd } \\
\text { with IA }\end{array}$ & $\begin{array}{l}\text { Disease status } \\
\text { IA Rx }\end{array}$ & $\begin{array}{l}\text { Age at IA } \\
\text { Rx (mo) }\end{array}$ \\
\hline$I^{a}$ & $U$ & 7 & No & B & No & No & OD & New Dx & 7 \\
\hline 2 & B & 5 & No & $D / D$ & Yes & $\begin{array}{l}\text { CEV(I0) Cy/T(3) } \\
\text { CV(3) }\end{array}$ & OD & Relapsed & 63 \\
\hline 3 & $U$ & 10 & No & B & No & No & OS & New Dx & 10 \\
\hline 4 & $U$ & 29 & No & $\mathrm{D}$ & No & No & OD & New Dx & 29 \\
\hline 5 & B & 40 & Yes & $\mathrm{D} / \mathrm{E}^{\mathrm{b}}$ & No & No & OD & New Dx & 41 \\
\hline 6 & B & 10 & No & $\mathrm{D} / \mathrm{D}$ & Yes & CEV(8) & OS & Refractory & 25 \\
\hline 7 & B & 33 & No & $\mathrm{E}^{\mathrm{b}} / \mathrm{D}$ & No & No & OS & New Dx & 33 \\
\hline 8 & B & 7 & No & $\mathrm{D} / \mathrm{D}$ & Yes & CEV(4) & OD & Refractory & 13 \\
\hline 9 & $U$ & 17 & No & $\mathrm{D}$ & Yes & No & OD & New Dx & 17.5 \\
\hline 10 & B & 4 & Yes & $\mathrm{D}$ & Yes & $\operatorname{CEV}(6)$ & OD & Refractory & 14 \\
\hline 10 & B & 4 & Yes & D & Yes & $\operatorname{CEV}(6)$ & OS & Refractory & 14 \\
\hline II & $U$ & 25 & No & $\mathrm{D}$ & No & No & OS & New Dx & 27 \\
\hline 12 & B & 8.5 & No & $\mathrm{C} / \mathrm{C}$ & Yes & $\operatorname{CEV}(6)$ & OD & Refractory & 15 \\
\hline 13 & B & 15 & No & $\mathrm{D}$ & Yes & No & OD & New Dx & 15 \\
\hline 13 & B & 15 & No & C & Yes & No & OS & New Dx & 15 \\
\hline 14 & B & 13 & No & $\mathrm{E}$ & No & No & OD & New Dx & 13 \\
\hline 14 & B & 13 & No & $E$ & No & No & OS & New Dx & 13 \\
\hline 15 & B & 0.5 & Yes & B & Yes & $\begin{array}{l}\mathrm{CV}(3) \\
\mathrm{CEV}(4) \\
\mathrm{Cy} / \mathrm{T}(3)\end{array}$ & OD & Refractory & 11 \\
\hline 15 & B & 0.5 & Yes & A & Yes & Same & OS & Refractory & 11 \\
\hline 16 & $U$ & 9 & Yes & B & Yes & No & OS & New Dx & 9.5 \\
\hline
\end{tabular}

Notes: apatient l's response can be seen in Figure I; 'group E eye enucleated at diagnosis; 'due to pathology results, systemic chemotherapy warranted.

Abbreviations: U, Unilateral; B, Bilateral; ICRB, International Classification of Retinoblastoma; RB, retinoblastoma; C, carboplatin; E, etoposide; V, vincristine; Cy, cyclophosphamide; T, topotecan; Dx, diagnosis; Rx, treatment; IA, intra-arterial; NED, no evidence of disease; AWD, alive with disease; mo, months.

for extraocular extension in addition to continued viable tumor on pathology despite three intra-arterial treatments. Ten eyes continued to receive local eye treatments after intra-arterial therapy.

Eleven of 20 eyes (55\%) were successfully spared radiotherapy or enucleation. In newly diagnosed eyes, 6/12 eyes $(50 \%)$ were spared radiotherapy or enucleation, and $5 / 8$ (63\%) refractory/relapsed eyes were spared radiotherapy or enucleation. Treatment success, defined by avoidance of radiotherapy and/or enucleation, was tabulated by international classification: 6/7 (86\%) in groups A-C and 5/13 $(38 \%)$ in groups D and E. All patients with local vitreous seeds responded to intra-arterial melphalan therapy and, of the patients with diffuse vitreous seeds, three failed intraarterial therapy secondary to vitreous seed progression. A demonstrative case (patient 1) before and after treatment is shown in Figure 1. All patients are alive at a median follow-up of 14.5 (range 1-29) months and no patients have developed metastatic disease. Overall, 11/16 patients are without evidence of disease and are off therapy.

\section{Toxicity}

Table 2 is a summary of local and systemic toxicity in the 16 patients who underwent 40 treatments of intra-arterial melphalan. There were no life-threatening or disabling complications from intra-arterial melphalan therapy. One patient had bronchospasm during microcatheterization of the ophthalmic

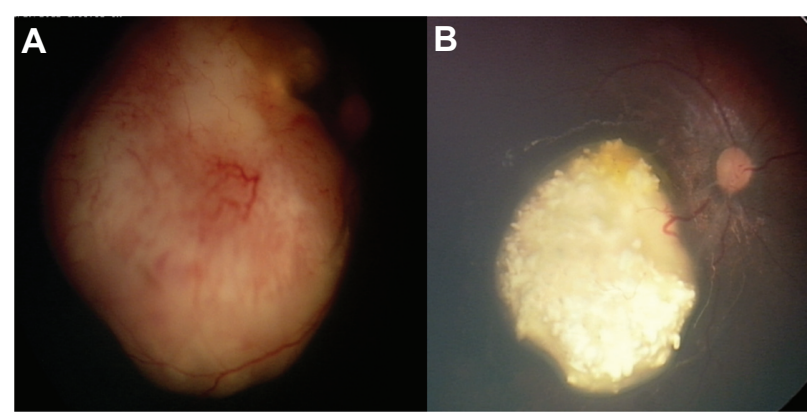

Figure I Tumor response to intra-arterial melphalan administration.

Notes: (Panel A) shows initial group B tumor at diagnosis in patient I, with tumor abutting the optic nerve. (Panel B) shows type I regression pattern one month after a single treatment with intra-arterial melphalan $(3 \mathrm{mg})$ without need for local laser or cryotherapy. 
Table I (Continued)

\begin{tabular}{|c|c|c|c|c|c|c|c|c|}
\hline Pt \# & $\begin{array}{l}\text { \# of IA } \\
R \mathbf{R x}\end{array}$ & $\begin{array}{l}\text { Wt at first } \\
\text { IA (kg) }\end{array}$ & $\begin{array}{l}\text { Dose of } \\
\text { melphalan }(\mathrm{mg})\end{array}$ & $\begin{array}{l}\text { Concurrent } \\
\text { local Rx }\end{array}$ & $\begin{array}{l}\text { Best response } \\
\text { to IA Rx }\end{array}$ & $\begin{array}{l}\text { Rx after IA } \\
\text { (\# of treatments) }\end{array}$ & Disease status & $\begin{array}{l}\text { Follow up after } \\
\text { first IA (mo) }\end{array}$ \\
\hline$\rho^{a}$ & 1 & 9.1 & 3 & No & Type I & No & NED off $R x$ & 4 \\
\hline 2 & 2 & 19.4 & $3,5 \mathrm{mg}$ & Yes & Type 4 & Radiation & NED, off Rx & 17 \\
\hline 3 & I & 8.5 & 4 & No & Type 2 & No & NED, off Rx & 20 \\
\hline 4 & I & 12.6 & 4 & No & Type I & Enucleation & NED, off Rx & 6 \\
\hline 5 & 3 & 20 & 5 & Yes & Decreased size & Radiation, enucleation & NED, off Rx & 9 \\
\hline 6 & I & 14.5 & 4 & Yes & Type 4 & Local $\operatorname{Rx}(7)$ & NED, off Rx & 15 \\
\hline 7 & I & 20 & 5 & No & $\begin{array}{l}\text { Unable to } \\
\text { assess }\end{array}$ & $\begin{array}{l}\text { Chemotherapyc } \\
\text { radiation }(b / I)\end{array}$ & NED, off Rx & 15 \\
\hline 8 & 4 & 10 & 4 & Yes & Type 4 & Local Rx (6) & AWD, ongoing $R x$ & 6 \\
\hline 9 & 5 & 9.5 & 4 & Yes & Type 3 & Local Rx (I0) & AWD, ongoing $R x$ & 17 \\
\hline 10 & 3 & 12.4 & 3 & Yes & Type 4 & Local Rx (7) & AWD, ongoing Rx & 14 \\
\hline 10 & 4 & 12.4 & $3,4 \mathrm{mg}$ & Yes & None & $\begin{array}{l}\text { Local Rx (5) } \\
\text { Enucleation }\end{array}$ & NED, off Rx & 14 \\
\hline II & 2 & 14 & 4 & No & None & Enucleation & NED, off Rx & 9 \\
\hline 12 & 4 & 13 & 4 & Yes & Type 3 & Local Rx (5) & NED, off Rx & 14 \\
\hline 13 & 3 & 10 & $3,4 \mathrm{mg}$ & Yes & Type I & Enucleation & NED, off Rx & 29 \\
\hline 13 & 2 & 10 & 3 & Yes & Type I & Local Rx (3) & NED, off Rx & 29 \\
\hline 14 & 3 & 10.6 & 3 & Yes & Type I & Enucleation & NED, off Rx & 22 \\
\hline 14 & 3 & 10.6 & 3 & No & Type I & $\begin{array}{l}\text { Chemotherapy } \\
\text { local } \operatorname{Rx}(2)\end{array}$ & NED, off Rx & 22 \\
\hline 15 & 2 & 8 & 3 & Yes & Type I and 4 & Local Rx (6) & NED, off Rx & 19 \\
\hline 15 & 4 & 8 & $3,4 \mathrm{mg}$ & Yes & Type 4 & $\begin{array}{l}\text { Radiation } \\
\text { local Rx (9) }\end{array}$ & AWD, ongoing Rx & 19 \\
\hline 16 & 1 & 9.7 & 3 & No & Type I & No & AWD, ongoing $R x$ & 1 \\
\hline
\end{tabular}

artery that was successfully treated with bronchodilators. However, as a result of transient wheezing post extubation, the patient was admitted for observation overnight and was discharged home the next day. Subsequent patients have received premedication with bronchodilators and no further events of bronchospasm have been observed. One patient had transient ophthalmic artery vasospasm with associated poor choroidal blush on angiography; this patient was admitted overnight for heparin therapy but had no later complications related to vision. Four patients had postoperative fever requiring overnight observation; one was diagnosed with acute otitis media and one had symptoms of upper respiratory tract infection.

Two patients developed local symptoms, including eyelid edema or eye irritation. One patient was found to have dacryohemorrhea (bloody tears). No patients were found to have cranial nerve palsy. Three patients were noted to have vitreous hemorrhage on follow-up examination under anesthesia. Patient 11 presented with an advanced group D eye and retinal detachment at 2 years of age. After the second intra-arterial melphalan treatment, the patient was noted to have a vitreous hemorrhage, which persisted on serial examinations and ultimately underwent enucleation for persistent disease and hemorrhage. Patient 14 presented with advanced group E eyes and bilateral retinal detachment at 13 months of age. This patient was also noted to have vitreous hemorrhage in one eye after the second intra-arterial treatment, and underwent an enucleation due to persistent vitreous hemorrhage limiting evaluation of the eye. Patient 8 presented with bilateral advanced group D eyes at 7 months of age. This patient was noticed to have a vitreous hemorrhage, with strands of blood noted after the fourth intra-arterial treatment. While this finding has persisted on follow-up examination, it does not obstruct retinal examination.

Two patients had grade 4 neutropenia, one of whom had an absolute neutrophil count of 300 at 15 days after intraarterial treatment and the other had an absolute neutrophil count of 350 at 12 days after intra-arterial treatment and required admission for fever. This patient was found to have central line-associated bacteremia with coagulase-negative 
Table 2 Toxicity of intra-arterial melphalan therapy

\begin{tabular}{ll}
\hline & Episodes (n) \\
\hline Eye toxicity & 2 \\
Eyelid edema/erythema & $\mathrm{I}$ \\
Dacryohemorrhea & 3 \\
Vitreous hemorrhage & $\mathrm{I}$ \\
Cataract & $\mathrm{I}$ \\
Retinal pigment and choroid atrophy & \\
Systemic toxicity & 4 \\
Postoperative fever & $2^{*}$ \\
Grade 4 neutropenia & $\mathrm{I}^{*}$ \\
Fever and neutropenia & \\
Procedure-related toxicity & $\mathrm{I}$ \\
Bronchospasm & $\mathrm{I}$ \\
Ophthalmic artery vasospasm & \\
Other & 0 \\
Blindness & 0 \\
Stroke &
\end{tabular}

Note: *Same patient with both fever and grade 4 neutropenia.

staphylococcus requiring removal of the line and treatment with antibiotics during admission and for 10 days after discharge. No patient required transfusions.

One patient developed a cataract in the eye treated with intra-arterial melphalan. Patient 14 underwent three intraarterial treatments along with concurrent local treatments, and 22 months after the first intra-arterial treatment, was found to have a focal cataract obstructing examination and will undergo cataract removal. This patient also received systemic chemotherapy because of pathology results from the other eye. One patient was found to have retinal pigment and choroid atrophy consistent with infarction on follow-up examination under anesthesia. After the fifth treatment with intra-arterial melphalan, patient 9 was found to have atrophy of the retinal pigment epithelium and choroid surrounding the inactive tumor, which has appeared stable on follow-up examinations.

Given the young age of our patients, most did not undergo formal vision testing after intra-arterial treatment with melphalan. Vision was followed based on reported history, response to light, extraocular movements, and ability to fix and follow. Patient 2 is older, and the last assessment of his vision was 20/50. Patient 14 has reported worsening vision which is due to a focal cataract.

\section{Radiation exposure from intra- arterial procedure}

The mean dose of radiation was $20.2 \pm 11.9 \mathrm{mGy}$ per eye per procedure. This mean dose was calculated based on 30 procedures.

\section{Discussion}

In this single-institution case series, we demonstrate that intra-arterial melphalan as a component of multimodal therapy spared 55\% (11/20) of eyes from enucleation or radiation. Patients with newly diagnosed disease had similar outcomes compared with patients having refractory/relapsed disease. Further, we confirmed previous findings demonstrating the tolerability of this approach and feasibility of administering this type of therapy at additional centers, with modest radiation exposure from the procedure.

Many groups have published reports on use of intra-arterial chemotherapy in refractory/relapsed cases. A Japanese group showed that eye preservation rates were highest, with International Classification groups $\mathrm{A}$ and $\mathrm{B}$ at $100 \%$ and $88 \%$, respectively, while groups $\mathrm{C}$ and $\mathrm{D}$ had rates of $65 \%$ and $45 \%$. Although group $\mathrm{E}$ had an eye preservation rate of only $30 \%$, this is significant because most group E eyes otherwise require enucleation. ${ }^{13}$ However, it is difficult to assess the overall efficacy of intra-arterial therapy, because this study included patients receiving concurrent therapies. Few reports reflect results for intra-arterial chemotherapy in newly diagnosed eyes. One group reported a 2-year ocular event-free survival (event defined as enucleation or external beam radiation therapy) of $81.7 \%$ at 2 years in patients treated with intra-arterial therapy as primary treatment, as compared with $58.4 \%$ in patients with a history of previous treatment failure. ${ }^{14}$ However, intra-arterial chemotherapy with melphalan alone or in combination with other intraarterial agents was used in that study.

We treated patients with newly diagnosed disease or refractory/relapsed disease from all international classification groups if the ocular oncologist determined that the eye was not amenable to local therapy. The outcome for newly diagnosed eyes $(50 \%)$ was lower than that for refractory/ relapsed eyes $(63 \%)$. While this finding is different from previous reports, it is likely that our newly diagnosed eyes had a worse response because of chance related to the small cohort and distribution of more advanced eyes in the newly diagnosed group (group $\mathrm{E}$ and scleral invasion on imaging warranting radiation). In addition, while only $25 \%$ of children with retinoblastoma are likely to present with bilateral disease, our cohort included 10/16 patients with bilateral retinoblastoma at diagnosis. It is possible that these children are more resistant to treatment.

Our results, when stratified according to classification group, are consistent with previous reports, with the exception of the single refractory group A eye which ultimately required external beam radiation therapy. Vitreous seeding 
in patients refractory to systemic chemotherapy has been reported to be difficult to control with intra-arterial therapy. ${ }^{15}$ However, two recent reports showed that advanced disease with subretinal and vitreous seeds can be controlled with intra-arterial therapy, although success rates are higher in treatment-naïve eyes. ${ }^{16,17}$ Difficulty controlling vitreous involvement in previously treated eyes was also seen in our cohort, with three patients who had vitreous seeding at diagnosis or relapse subsequently receiving radiation for progression of vitreous seeds despite intra-arterial treatment. Two of these patients had previously received systemic chemotherapy. The third patient could not be evaluated for response to intra-arterial treatment because this patient had also received systemic chemotherapy as a result of high-risk pathologic features (choroidal and optic nerve invasion) found at enucleation of the contralateral eye. In some centers, intravitreal chemotherapy has also been used in the setting of persistent vitreous seeds. ${ }^{18,19}$ While most studies have involved advanced disease, Abramson et al reported ocular event-free survival of $96 \%$ in group B and C cases. ${ }^{20}$ For advanced cases, reports of $45 \%-100 \%$ success have been reported for group D and 0\%-33\% for group E. ${ }^{13,17,21,22}$ Other groups have classified patients using the Reese Ellsworth classification, with advanced eyes grouped as V described as having ocular event-free survival of up to $66.5 \%{ }^{14}$

Our patients were treated with melphalan as a single agent, as in a number of other reports. ${ }^{17,22-24}$ However, those groups focused their treatment on patients with either advanced retinoblastoma or refractory cases. Other reports have described combination intra-arterial chemotherapy with use of melphalan together with carboplatin and/or topotecan for a broad range of indications, including newly diagnosed and refractory cases, as well as a range of disease extent by international classification. ${ }^{12-14,25}$ While we chose to use single-agent melphalan as the most widely studied agent for our initial approach, the optimal chemotherapy drug or drug combination for use in intra-arterial therapy must be determined.

Our dosing was based on data reported by Gobin et al, who used doses based on age, given that this generally correlates with globe size. ${ }^{14}$ Other institutions report similar dosing practices. ${ }^{26}$ Some investigators have reported superior results using higher doses of melphalan. For instance, Peterson et al reported better results in patients with advanced and refractory retinoblastoma when a dose of $7.5 \mathrm{mg}$ was used. $^{22}$ These patients had group D eyes and disease refractory to systemic chemotherapy. Seven of ten patients treated initially at 3-5 mg were given $7.5 \mathrm{mg}$ at the next treatment due to lack of response. Four of 17 eyes required enucleation; all enucleated eyes were treated initially at the lower dose of 3-5 mg. In this group, there were two cases of neutropenia and one case of fever reported, but it was unclear if toxicity was worse with the higher doses of melphalan. In our series, we had two patients (5\%) with grade 4 neutropenia (absolute neutrophil count $<500$ ) as defined by Common Terminology Criteria for Adverse Events version 4.0. Other groups have reported similar rates of neutropenia. Abramson et al also reported one episode of grade 4 neutropenia and nine episodes of grade 3 neutropenia out of 75 infusions. ${ }^{25}$ This same group also reported 21/255 episodes of grade 3 neutropenia and $8 / 255$ episodes of grade 4 neutropenia in another report. ${ }^{14}$

As expected, based on previous reports, acute side effects such as vasospasm and bronchospasm were noted, although no serious complications resulted. We had three cases of transient local eye changes, irritation, eyelid edema, and dacryohemorrhea, and Suzuki et al also reported transient eye changes in some patients and transient bronchospasm in one patient. ${ }^{13}$ Abramson et al reported adverse ocular findings from these procedures, such as transient lid edema, forehead hyperemia, and loss of lashes. ${ }^{25}$ Three eyes in our series developed a vitreous hemorrhage, although only two that obstructed examination and one eye with strands of blood in the vitreous, and all found after intra-arterial treatment. This is comparable with the findings of Peterson et al who noted $4 / 26$ cases (15\%) and Vajzovic et al who noted $3 / 12(25 \%)$ episodes of vitreous hemorrhage in advanced retinoblastoma cases that ultimately led to enucleation. ${ }^{22,24}$ While the cause of vitreous hemorrhage is unknown, it appeared in patients with advanced tumors, both in our data series and that of Peterson et al. ${ }^{22}$ We found one eye not treated with external beam radiation that has developed a cataract. While this is reported rarely, Gobin et al noted three eyes with cataracts, two of which had previously been treated with radiation therapy. ${ }^{14}$ We also found one eye with retinal pigment and choroid atrophy noted after the fifth treatment with intra-arterial melphalan. Retinal pigment epithelial changes and choroid infarction are recognized complications of intra-arterial chemotherapy, and have been described by a number of centers..$^{23,26,27}$ Muen et al noted $7 / 15(47 \%)$ cases of significant retinal pigment epithelium atrophy. ${ }^{23}$ Shields et al reported retinal pigment epithelial mottling in nine cases $(56 \%)$ that later evolved to choroidal atrophy in five cases $(31 \%) .{ }^{27}$

Radiation exposure from fluoroscopy is a concern in this population of very young children who have an increased lifelong predisposition to secondary cancers. Previously reported 
radiation exposure to the treated eye has ranged widely, from $5.55 \mathrm{mGy}$ to $191 \mathrm{mGy}^{28,29}$ Institutions performing intra-arterial chemotherapy should evaluate their procedures and radiation doses to ensure that the lowest possible doses are being used. Our radiation dose of $20.2 \mathrm{mGy}$ per eye per procedure is comparable with previous reports, and is well below the reported doses at which the risk of cataract formation (500 mGy) and sarcoma formation (>5000 mGy) are increased. ${ }^{29,30}$

Inherent limitations to our study include the small number of patients and its retrospective nature. While our data add to the literature regarding acute toxicities related to intraarterial melphalan therapy, long-term follow-up studies will be important to evaluate any potential risks of secondary malignancy.

In conclusion, we have shown that intra-arterial administration of melphalan is feasible in patients with newly diagnosed or refractory/relapsed retinoblastoma with a range of ocular involvement. In our series, 11/20 eyes were spared enucleation or radiation therapy. Prospective long-term follow-up studies are needed to evaluate the late effects of this approach, including an assessment of treatment impact on visual acuity in a formalized way. Further clarification of the optimal melphalan dose and the role of intra-arterial combination chemotherapy regimens is needed.

\section{Acknowledgments}

We are grateful to our nurses, Ilana Withop and Jeanne Scanlon, and research assistants, Tia Cole and Keith Sharee, for their assistance in managing these patients and compiling information for this report. We also appreciate the support received from the chair endowed by Mildred V Strouss, the Campini Foundation, and the Alex Scott Lemonade Foundation.

\section{Disclosure}

The authors report no conflicts of interest in this work.

\section{References}

1. Young JL, Smith MA, Roffers SD, et al. Retinoblastoma. Available from: http://seer.cancer.gov/publications/childhood/retinoblastoma.pdf. Accessed March 16, 2013.

2. Pizzo PA, Poplack DG. Principles and Practice of Pediatric Oncology, 5th ed. Philidelphia, PA: Lippincott Williams \& Wilkins; 2006.

3. Broaddus E, Topham A, Singh AD. Survival with retinoblastoma in the USA: 1975-2004. Br J Ophthalmol. 2009;93:24-27.

4. Kleinerman RA, Tucker MA, Tarone RE, et al. Risk of new cancers after radiotherapy in long-term survivors of retinoblastoma: an extended follow-up. J Clin Oncol. 2005;23:2272-2279.

5. Murphree AL. Intraocular retinoblastoma: the case for a new group classification. Ophthalmol Clin North Am. 2005;18:41-53.
6. Shields CL, Shields JA. Retinoblastoma management: advances in enucleation, intravenous chemoreduction, and intra-arterial chemotherapy. Curr Opin Ophthalmol. 2010;21:203-212.

7. Reese AB, Hyman G, Tapley N, Forrest AW. The treatment of retinoblastoma by X-ray and triethylene melamine. AMA Arch Ophthalmol. 1958;60:897-906.

8. Kiribuchi M. Retrograde infusion of anti-cancer drugs to ophthalmic artery for intraocular malignant tumors. Nippon Ganka Gakkai Zasshi. 1966;70:1829-1833. Japanese.

9. Mohri M. The technique of selective ophthalmic arterial infusion for conservative treatment of recurrent retinoblastoma. Keio Igaku. 1993;70:679-687. Japanese.

10. Kaneko A. Japanese contributions to ocular oncology. Int J Clin Oncol. 1999;4:321-326.

11. Yamane T, Kaneko A, Mohri M. The technique of ophthalmic artery infusion therapy for patients with intraocular retinoblastoma. Int J Clin Oncol. 2004;9:69-73.

12. Abramson DH, Dunkel IJ, Brodie SE, Kim JW, Gobin YP. A Phase I/II study of direct intraarterial (ophthalmic artery) chemotherapy with melphalan for intraocular retinoblastoma: initial results. Ophthalmology. 2008;115:1398-1404.

13. Suzuki S, Yamane T, Mohri M, Kaneko A. Selective ophthalmic arterial injection therapy for intraocular retinoblastoma: the long-term prognosis. Ophthalmology. 2011;118:2081-2087.

14. Gobin YP, Dunkel IJ, Marr BP, Brodie SE, Abramson DH. Intra-arterial chemotherapy for the management of retinoblastoma: four-year experience. Arch Ophthalmol. 2011;129:732-737.

15. Kim J, Do H, Egbert P. Enucleated eyes after failed intra-arterial infusion of chemotherapy for unilateral retinoblastoma: histopathologic evaluation of vitreous seeding. Clin Ophthalmol. 2011;5:1655-1658.

16. Abramson DH, Marr BP, Dunkel IJ, et al. Intra-arterial chemotherapy for retinoblastoma in eyes with vitreous and/or subretinal seeding: 2-year results. Br J Ophthalmol. 2012;96:499-502.

17. Shields CL, Bianciotto CG, Jabbour P, et al. Intra-arterial chemotherapy for retinoblastoma: Report No 1, control of retinal tumors, subretinal seeds, and vitreous seeds. Arch Ophthalmol. 2011;129:1399-1406.

18. Munier F, Gaillard MC, Balmer A, et al. Intravitreal chemotherapy for vitreous disease in retinoblastoma revisited: from prohibition to conditional indications. Br J Ophthalmol. 2012;96:1078-1083.

19. Ghassemi F, Shields $\mathrm{Cl}$. Intravitreal melphalan for refractory or recurrent vitreous seeding from retinoblastoma. Arch Ophthalmol. 2012;130:1268-1271.

20. Abramson DH, Marr BP, Brodie SE, Dunkel I, Palioura S, Gobin YP. Ophthalmic artery chemosurgery for less advanced intraocular retinoblastoma: five year review. PLoS One. 2012;7:e34120.

21. Shields CL, Kaliki S, Shah SU, et al. Minimal exposure (one or two cycles) of intra-arterial chemotherapy in the management of retinoblastoma. Ophthalmology. 2012;119:188-192.

22. Peterson EC, Elhammady MS, Quintero-Wolfe S, Murray TG, Aziz-Sultan MA. Selective ophthalmic artery infusion of chemotherapy for advanced intraocular retinoblastoma: initial experience with 17 tumors. J Neurosurg. 2011;114:1603-1608.

23. Muen WJ, Kingston JE, Robertson F, Brew S, Sagoo MS, Reddy MA. Efficacy and complications of super-selective intra-ophthalmic artery melphalan for the treatment of refractory retinoblastoma. Ophthalmology. 2012;119:611-616.

24. Vajzovic LM, Murray TG, Aziz-Sultan MA, et al. Supraselective intraarterial chemotherapy: evaluation of treatment-related complications in advanced retinoblastoma. Clin Ophthalmol. 2010;5:171-176.

25. Abramson DH, Dunkel IJ, Brodie SE, Marr B, Gobin YP. Supraselective ophthalmic artery chemotherapy as primary treatment for retinoblastoma (chemosurgery). Ophthalmology. 2010;117:1623-1629.

26. Venturi C, Bracco S, Cerase A, et al. Superselective ophthalmic artery infusions of melphalan for intraocular retinoblastoma: preliminary results from 140 treatments. Acta Ophthalmol. January 23, 2012. [Epub ahead of print.] 
27. Shields CL, Bianciotto CG, Jabbour P, et al. Intra-arterial chemotherapy for retinoblastoma: Report No 2, treatment complications. Arch Ophthalmol. 2011;129:1407-1415.

28. Gobin YP, Rosenstein LM, Marr BP, Brodie SE, Abramson DH. Radiation exposure during intra-arterial chemotherapy for retinoblastoma. Arch Ophthalmol. 2012;130:403-404.
29. Vijayakrishnan R, Shields CL, Ramasubramanian A, Emrich J, Rosenwasser R, Shields JA. Irradiation toxic effects during intraarterial chemotherapy for retinoblastoma. Arch Ophthalmol. 2010;128: 1427-1431.

30. Wong LF, Boice JD, Abramson DH, et al. Cancer incidence after retinoblastoma. JAMA. 1997;278:1262-1267.

\section{Publish your work in this journal}

Clinical Ophthalmology is an international, peer-reviewed journal covering all subspecialties within ophthalmology. Key topics include: Optometry; Visual science; Pharmacology and drug therapy in eye diseases; Basic Sciences; Primary and Secondary eye care; Patien Safety and Quality of Care Improvements. This journal is indexed on

Submit your manuscript here: http://www.dovepress.com/clinical-ophthalmology-journal

\section{Dovepress}

PubMed Central and CAS, and is the official journal of The Society of Clinical Ophthalmology (SCO). The manuscript management system is completely online and includes a very quick and fair peer-review system, which is all easy to use. Visit http://www.dovepress.com/ testimonials.php to read real quotes from published authors. 